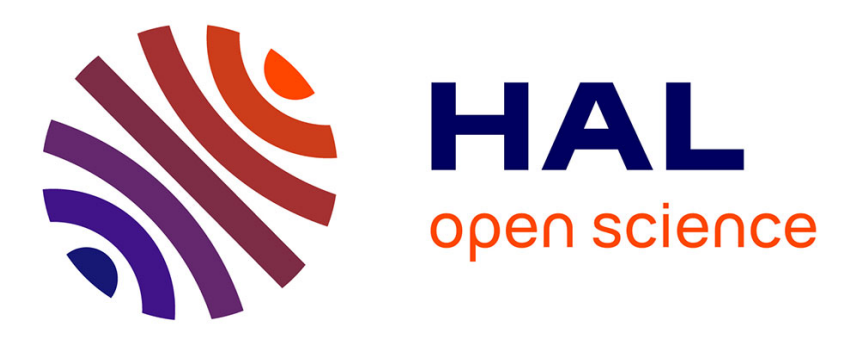

\title{
An Assistive Explicit Model Predictive Control Framework for a Knee Rehabilitation Exoskeleton
}

\author{
Ines Jammeli, Ahmed Chemori, Huiseok Moon, Salwa Elloumi, Samer
} Mohammed

\section{- To cite this version:}

Ines Jammeli, Ahmed Chemori, Huiseok Moon, Salwa Elloumi, Samer Mohammed. An Assistive Explicit Model Predictive Control Framework for a Knee Rehabilitation Exoskeleton. IEEE/ASME Transactions on Mechatronics, 2022, 27 (5), pp.3636-3647. 10.1109/TMECH.2021.3126674 . lirmm03495187

\author{
HAL Id: lirmm-03495187 \\ https://hal-lirmm.ccsd.cnrs.fr/lirmm-03495187
}

Submitted on 20 Dec 2021

HAL is a multi-disciplinary open access archive for the deposit and dissemination of scientific research documents, whether they are published or not. The documents may come from teaching and research institutions in France or abroad, or from public or private research centers.
L'archive ouverte pluridisciplinaire HAL, est destinée au dépôt et à la diffusion de documents scientifiques de niveau recherche, publiés ou non, émanant des établissements d'enseignement et de recherche français ou étrangers, des laboratoires publics ou privés. 


\title{
An Assistive Explicit Model Predictive Control Framework for a Knee Rehabilitation Exoskeleton
}

\author{
Ines Jammeli, Ahmed Chemori Senior Member, IEEE, Huiseok Moon, Salwa Elloumi and Samer Mohammed \\ Senior Member, IEEE
}

\begin{abstract}
This study focuses on the control of an actuated knee joint orthosis. The proposed solution is a novel model predictive control framework dedicated to assistive and rehabilitation purposes. This framework includes $(i)$ an exact inputto-state feedback linearization, (ii) a model predictive controller (MPC or EMPC), considering input/output constraints, (iii) a least-squares dynamic parameters identification, (iv) a nonlinear disturbance observer for the estimation of the wearer's torque, (v) a Lyapunov-based stability analysis of the resulting closedloop system, and (vi) a reference trajectory generator. The proposed framework has been validated via real-time experiments performed on three healthy subjects wearing the knee joint orthosis. Various experimental scenarios have been considered, including assistive and resistive rehabilitation tasks in a sitting position and walking with normal/abnormal gait patterns. The obtained results indicate the efficiency of the proposed predictive controllers with respect to a conventional PID controller in terms of tracking performance, required torque, and wearer comfort.
\end{abstract}

Index Terms-Assistive Robotics, Knee joint powered orthosis, Model predictive control, Rehabilitation

\section{INTRODUCTION}

W ITH recent medical advances, life expectancy increases steadily. According to the World Health Organization, the world population aged 60 years and older is expected to reach a total of 2 billion by 2050; however, it was approximately 900 million in 2015 [1]. This population is exposed to health risks caused by weakened muscle strength, which hinders their ability to walk as frequently as normal and adversely affects their walking stability, thereby making them dependent on others. Consequently, the aging of the population and the physical deterioration of the elderly have become a global socioeconomic problem [2]. This issue calls for considerable attention on how to assist this population, as well as people with lower and/or upper-limb pathologies in their daily life, especially regarding mobility and autonomy [3].

One of the best remedies for reduced mobility is rehabilitation. Conventional intensive therapies are usually adopted in

Manuscript received June 10, 2020; revised July 99, 9999.

This work did not receive any financial support. (Corresponding Author: Ahmed Chemori).

I. Jammeli (e-mail: jammeli.ines@gmail.com) and S. Elloumi (e-mail: salwa.elloumi@gmail.com) are with LSA, EPT, University of Carthage BP. 743, 2078 La Marsa, Tunis, Tunisia.

A. Chemori is with LIRMM, University of Montpellier, CNRS, Montpellier, France (e-mail: Ahmed.Chemori@lirmm.fr).

H. Moon and S. Mohammed are with LISSI, EA 3956 UPEC, 122 rue Paul Armangot, 94400 Vitry-Sur-Seine, France (e-mail: samer.mohammed@upec.fr). clinical centers to help people recover their voluntary movements. However, the problem with these therapies is that they are only effective when they are intensive [4]. Furthermore, repetition is a key element in this case, which allows the brain to reprogram the motion sequence. Therefore, this process is time and capital intensive, and requires the strength of both the patient and therapist. However, intensive long-term rehabilitation therapy is not always an option owing to its expensive cost, and the insufficiency of qualified staff.

To reduce the burden on care services, several initiatives have been set up to promote assistive technologies, such as the promising technologies based on wearable robots. These devices are mechatronic systems, equipped with sensors and actuators, and embodied by the human upper and/or lowerlimbs, which provide the following functions: (i) augmenting physical human capabilities at upper/lower-limbs, (ii) assisting people with reduced mobility for achieving daily living activities, and (iii) automating the rehabilitation of human joints and muscles to recover and improve the control of the wearer's limbs [5]. This considerable focus on wearable robots can be explained by their ability to reproduce repetitive tasks that require strength and robustness. They can autonomously perform these tasks faster than therapists, with a better level of accuracy, without getting tired, and without requiring a third party. Hence, they may promote a reduction in patient fatigue. They enable long training sessions with optimal consistency, as well as measurements for the user to track the desired gait patterns, which may help to accelerate the rehabilitation process [6].

Sitting and standing up movements are essential for most human activities. Given their importance, several wearable robots have been developed to help weakened people perform these movements. Shepherd et al. [7] designed a knee exoskeleton using a torque-controllable series elastic actuator capable of providing the torques and speeds required for sitto-stand. In [8], the authors proposed an intention-based active impedance control (AIC) strategy applied to a lower-limb exoskeleton for wearers with a partial loss of their lower-limb muscular strength. In [9], the authors designed an exoskeleton to assist the wearer during sit to stand tasks by delivering assistance-as-needed to patients or elderly people with muscle weakness. In [10], a trajectory generator and an impedance controller were designed to calculate the required torques for a knee exoskeleton to assist the wearer in moving along a predicted trajectory while allowing the wearer to partly control the speed of the sit-to-stand movement. If sitting and standing up movements are essential for most human 
activities, walking is even more essential, and it plays a key role in everyday life. Losing the ability to walk presents a real challenge, which makes the patient dependent on others. To address this challenge and enable people with reduced mobility to recover their independence, significant attention has been given to exoskeletons. To assist people while walking, Wang et al. [11] developed a highly back-drivable, partial-assist knee orthosis designed to improve excessive shear forces, reduce joint misalignment, and minimize the distal weight. In [12], the authors presented the design of a partial-assist knee orthosis by improving its back-drivability, weight, and size. They reduced the motor winding temperature and enabled higher output torques by adopting an encapsulation technology. In [13], the authors presented a task-invariant and usercooperative approach, known as energy shaping, to provide assistance by augmenting body energetics. Although some of the aforementioned controllers provide fairly satisfactory performances, none of them can provide optimal control while considering the system constraints. In this study, we focus on the control of exoskeleton intelligently communicating and sensitive to intention (EICOSI) system, an active lower-limb orthosis acting on the knee joint level, developed for assistive and rehabilitation purposes. This system has a simple structure, is easy to don and doff, and practical for people suffering from knee joint impairments. Various control approaches have been proposed for EICOSI such as PID control, adaptive control [14] [15], and high-order sliding mode control [3].

In this study, a novel model predictive control framework (illustrated in Fig. 1), dedicated to assistive and rehabilitation purposes, is proposed. This framework includes (i) an exact input-to-state feedback linearization, (ii) a model predictive controller (MPC) considering input/output constraints, (iii) a least-squares dynamic parameters identification, (iv) a nonlinear disturbance observer (NDO) for the estimation of the wearer's torque, $(v)$ a Lyapunov-based stability analysis of the resulting closed-loop system, and (vi) a reference trajectory generator.

Although MPC has several advantages in comparison with conventional controllers, to the best of our knowledge, the proposed controller has not been used in an assistive context with actuated orthosis. This control allows desired performances to be expressed in a single cost function that can be easily adjusted according to each user. Moreover, the tracking error is guaranteed along with the smoothness of the orthosis behavior ensured by the minimization of the cost function in the MPC control formulation. This smoothness could have a considerable and favorable impact on the wearer's comfort. Furthermore, the tracking error shows the ability of the controller to follow a desired trajectory while taking into account external perturbations and uncertainties. Providing satisfactory tracking performances as prescribed by therapist doctors within a rehabilitation process will ensure that the rehabilitation purposes in terms of muscle reinforcement and joint range of movement enhancement are met. The MPC formulation is also characterized by its ability to implicitly consider constraints on the control inputs (torques), as well as on the states and outputs (position, velocity, and acceleration), which may ensure the safety of the wearer, and the stability of the overall control strategy. In addition, MPC has an anticipation feature, which enables it to anticipate a forthcoming change in the desired behavior of the system to be controlled. MPC can also be considered a robust control that can compensate for parametric uncertainties resulting from a poor identification process, or for external disturbances such as the exerted human torques or disturbances originating from the interaction with the ground. The proposed framework has been validated via real-time experiments performed on healthy subjects (a) in a sitting position while asked to exert an assistive/resistive effort, and (b) during a walking activity while considering the interaction with the ground, and following a reference walking pattern generated by the proposed trajectory generator. The exerted human torque and the effect of the ground interaction during the stance phase were estimated using the proposed NDO. The rest of the paper is organized as follows: A description of the EICOSI active lower-limb orthosis is presented in section II The proposed MPC approaches are discussed in section III Section IV is dedicated to the proposed NDO, as well as the proposed walking trajectory generator. The resulting closedloop system stability analysis is discussed in section V Section VI gathers all the common methods between the conducted experiments and illustrates the experimental protocol. The obtained experimental results are presented and discussed in section VII Finally, section VIII provides some concluding remarks, and projections for future works.

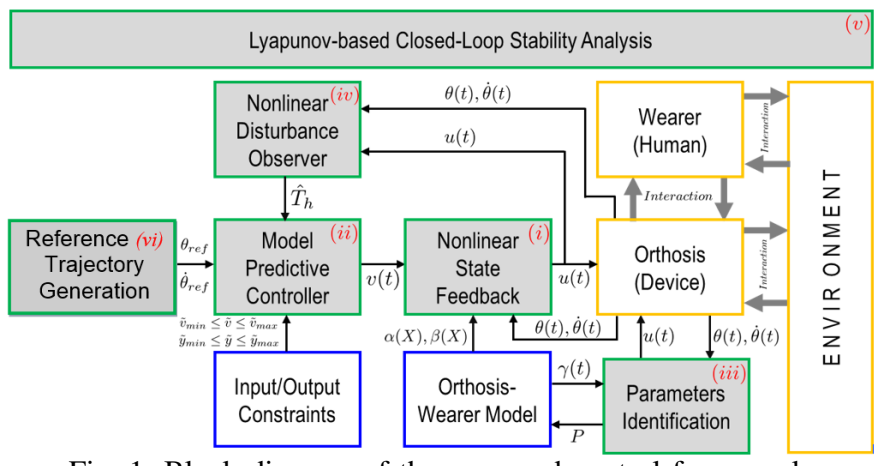

Fig. 1: Block diagram of the proposed control framework.

\section{EICOSI ACTIVE ORTHOSIS}

\section{A. Description and modelling of EICOSI orthosis}

EICOSI active orthosis is a single DoF prototype adopted in this study to assist the wearer at the knee joint level for flexion/extension movements in a sitting position and during a walking activity. Fig. 2 shows the subject (a) in a sitting rehabilitation position and (b) walking on a treadmill while wearing the actuated orthosis. EICOSI comprises two segments attached separately to the wearer's thigh and shank using appropriate braces. The shank-foot of the wearer is considered as the single rigid segment, and it freely moves around the knee joint. The orthosis is driven using an EC-4pole Maxon 200W motor controlled by an ESCON 50/8 driver. A real-time communication with FPGA card is established. To guarantee an efficient and portable system, as well as relatively high output torque, a compact transmission system is designed using a gear motor, ball screw, transmission belt, and cable 
drive. The reduction ratio from the motor to the joint side is $264: 1$. The actuation system can deliver a torque up to

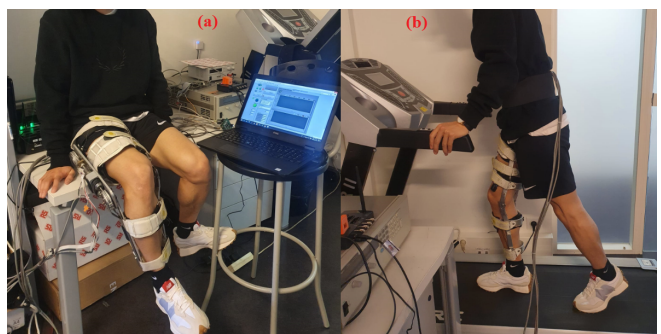

Fig. 2: Images of the subject wearing the EICOSI exoskeleton (a) in a sitting rehabilitation position, and (b) walking on a treadmill.

18 N.m, which is estimated using a current sensor. The motor is equipped with an incremental encoder to measure its angular position, which is used to compute the knee joint angle. The angular velocity and acceleration are obtained by numerical derivatives (first and second-order respectively) of the angular position. The thigh angle is measured using an IMU (inertial measurement unit: MTw Awinda by $\mathrm{Xsens}^{\Theta}$ ) attached to the thigh brace, rather than the user's shank, to obtain less noise from ground interaction, and facilitate the measurements at the sitting position. The experiments were performed using the LabView software. This active orthosis enables its user to perform flexion/extension movements with a relative angle ranging from $0^{\circ}$ to $135^{\circ}$, where the latter corresponds to the maximum knee joint-orthosis flexion and the former to the full knee joint-orthosis extension. For dynamic modelling, the application of Newton's second law gives:

$J \ddot{\theta}=\sum_{i} \tau_{i}=\tau_{e}+\tau_{h}-\tau_{g}-\tau_{f}-\tau_{l}$, where $\tau_{e}, \tau_{h}$, $\tau_{g}$, and $\tau_{f}$ represent the exoskeleton torque, human torque, gravitational toque, and friction torque, respectively. The latter primarily comprises static and viscous frictions, expressed by $A \operatorname{sign}(\dot{\theta}(t))+B(\dot{\theta}(t))$, where $A$ and $B$ respectively represent the coefficients of static and viscous friction torques of the entire system, including the human leg and the exoskeleton. It is noteworthy that the adopted friction model is advantageous because it is linear in its parameters $A$ and $B . \tau_{l}$ represents the lumped torque triggered by the other body segments and the eventual external forces. $J$ is the inertia of the human lower limb wearing the active orthosis. The wearer's shank and the embodied actuated orthosis system can be dynamically modeled by the following second-order equation:

$$
J \ddot{\theta}=-T_{g} \sin \left(\theta-\theta_{t}\right)-A \operatorname{sign}(\dot{\theta})-B(\dot{\theta})+\tau_{e}+\tau_{h}-\tau_{l}
$$

where $\theta, \dot{\theta}$, and $\ddot{\theta}$ represent the angular position, velocity, and acceleration of the entire lower part system (including the wearer's shank and the active orthosis lower part), respectively. $\theta_{t}$, and $\dot{\theta}_{t}$ are the angular position and velocity of the upper part (including the wearer's thigh and the upper part of the orthosis [16]), respectively. In this work, we are interested in the application of the proposed MPC-based framework on EICOSI orthosis, both in a sitting rehabilitation position and for a walking activity. The human gait cycle can be categorized into two different phases as illustrated in Fig. 3 The stance phase, accounting for $60 \%$ of the cycle, during which the foot is placed on the ground, and the swing phase, accounting for $40 \%$ of the cycle, during which the foot is no longer in contact with the ground. In the swing phase, the lumped torque $\tau_{l}$ can

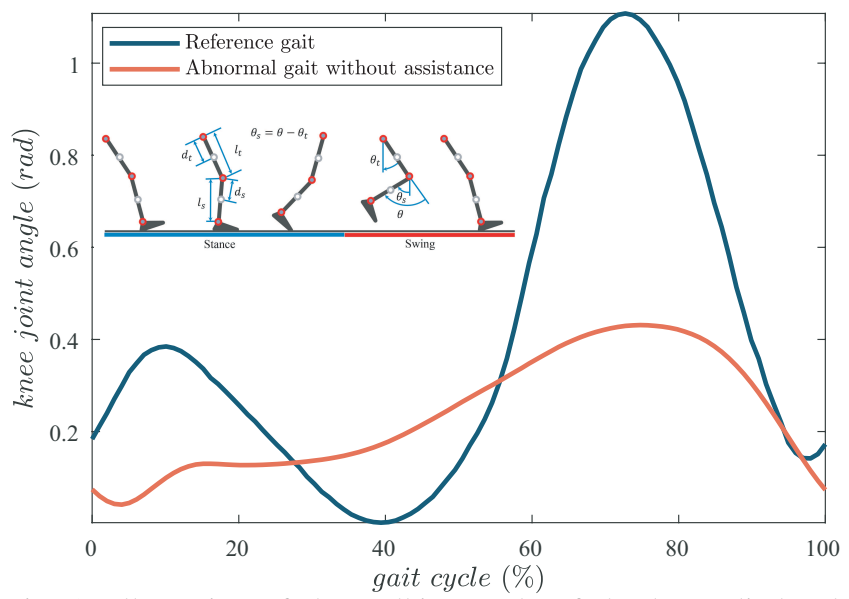

Fig. 3: Illustration of the walking cycle of the lower limb while wearing the exoskeleton, and with the generated walking trajectory (for both reference and abnormal gaits).

be expressed by the following equation [17]:

$$
\tau_{l}=m_{s} d_{s} l_{t}\left(\ddot{\theta}_{t}(t) \cos (\theta(t))-\dot{\theta}_{t}^{2}(t) \sin (\theta(t))\right)
$$

where $m_{s}, l_{t}$, and $d_{s}$ represent the lumped mass of the human shank and the corresponding orthosis segment, human thigh length, and length of the human shank part from the knee joint center to the center of mass of the shank-foot, respectively. In addition, $\dot{\theta}_{t}$ and $\ddot{\theta}_{t}$ are the velocity and acceleration of the upper part, respectively. For the stance phase, the contact forces with the ground are considered external perturbations. Because the studied active orthosis has only one degree of freedom, and does not include an ankle actuator, the ground does not interact directly with the exoskeleton; hence, the reaction forces could be considered as external disturbances to the system. In this case, the effect of the ground contact can be considered within the torque estimated by the proposed NDO.

\section{B. Dynamic identification of parameters}

The proposed control framework (cf. Fig. 1) includes a nonlinear state feedback and an MPC, both of which are modelbased control strategies. Consequently, the dynamic model of the system is significantly beneficial in the performance of the proposed control scheme. To minimize modeling errors, an identification process is performed as follows. We ask the subject to wear the exoskeleton, stay in a sitting position, and be passive (i.e. not generating any voluntary movement) during the identification process. Because the human-orthosis is considered to be a single system, we assume that $\tau_{h}, \tau_{l}, \dot{\theta}_{t}$, and $\ddot{\theta}_{t}$ are zero and that $\theta_{t}=\frac{\pi}{2}$. Hence, the inverse dynamic model can be represented by the following equation:

$$
\tau_{e}=J \ddot{\theta}-T_{g} \cos (\theta)+A \operatorname{sign}(\dot{\theta})+B \dot{\theta}
$$

which can be reformulated as affine in the parameters:

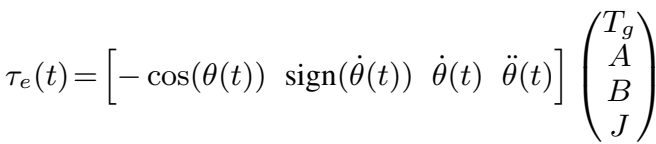

$$
\begin{aligned}
& =\gamma^{T}(t) P
\end{aligned}
$$


with $\gamma^{T}(t)=[-\cos (\theta(t)) \operatorname{sign}(\dot{\theta}(t)) \dot{\theta}(t) \ddot{\theta}(t)]$ and $P=$ $\left[\begin{array}{llll}T_{g} & A & B & J\end{array}\right]^{T}$. The identification algorithm is based on the adoption of the inverse model (4), which is linear in the dynamic parameters of the system. It enables the estimation of these parameters, providing either a measurement or an estimation of the joint torque $\tau_{e}$, as well as the joint position $\theta$, velocity $\dot{\theta}$, and acceleration $\ddot{\theta}$. Let us define $T=\left[\begin{array}{llll}\tau_{e}(1) & \tau_{e}(2) & \ldots & \tau_{e}(n)\end{array}\right]^{T}$, and $\Upsilon=$ $\left[\gamma^{T}(1) \gamma^{T}(2) \ldots \gamma^{T}(n)\right]^{T}$, with $n$ as the number of samples. The parameters of our system, i.e $T_{g}, A, B$, and $J$ are computed by minimizing the error:

$$
\varepsilon(t)=\tau_{e}(t)-\gamma^{T}(t) P
$$

via the optimization of the following cost function:

$$
\psi(P)=\frac{1}{n} \sum_{t=1}^{n} \varepsilon^{2}(t)
$$

The optimal parameters can be obtained by zeroing the term $\frac{d \psi(P)}{d P}$. Computing $P=\left(\Upsilon^{T} \Upsilon\right)^{-1} \Upsilon^{T} T$, facilitates the identification of the studied system parameters [18]. Different series of various tests were performed using a basic proportional controller while applying chirp signals as inputs. For each test, a different amplitude was used. This enables us to obtain the system response to several amplitudes and frequencies. Each parameter has been computed as the average of the obtained values from different tests, and are summarized in TABLE [.

TABLE I: Identified parameters of EICOSI orthosis for the three participating subjects.

\begin{tabular}{ccccc}
\hline Parameter & Symbol & S1 & S2 & S3 \\
\hline Static friction coefficient $($ N.m $)$ & $A$ & 2.0082 & 1.2067 & 0.7613 \\
Viscous friction coefficient $N . m . s$. rad $^{-1}$ & $B$ & 1.713 & 3.238 & 2.4539 \\
Inertia Kg.m m $^{2}$ & $J$ & 0.4325 & 0.2594 & 0.2525 \\
Gravity torque $($ N.m $)$ & $T_{g}$ & 9.4199 & 10.4741 & 3.4379 \\
\hline
\end{tabular}

\section{Input-to-state feedback linearization}

The proposed MPC approach is a discrete-time controller based on a linear model of the system. However, the studied active orthosis has a continuous nonlinear model; therefore, it should be linearized and discretized to integrate it into the MPC controller. The approximated linearization of the EICOSI system (1) is not feasible owing to the existence of the term $\operatorname{sign}(\dot{\theta})$, which gives it a highly nonlinear feature. Consequently, we propose the use of an exact input-to-state linearization approach, which can transform the nonlinear model into an equivalent linear alternative via a change of variables and a suitable nonlinear feedback. Let us consider the nonlinear state feedback:

$$
\tau_{e}=\alpha(X)+\beta(X) v
$$

By selecting the adequate control input $\tau_{e}$, this approach facilitates the emergence of a linear input-output map between the linearized input $v$ and the output $y$. For the sitting position, we set:

$$
\left\{\begin{array}{l}
\alpha(X)=-T_{g} \cos (\theta)+A \operatorname{sign}(\dot{\theta})+B(\dot{\theta})-\tau_{h} \\
\beta(X)=J \\
X=\left[\begin{array}{ll}
x_{1} & x_{2}
\end{array}\right]^{T}=[\theta \quad \dot{\theta}]^{T}
\end{array}\right.
$$

The nonlinear state feedback $\tau_{e}$ can then be expressed as:

$$
\tau_{e}(t)=-T_{g} \cos (\theta)+A \operatorname{sign}(\dot{\theta})+B(\dot{\theta})-\tau_{h}+J v .
$$

For the standing position, we set:

$$
\left\{\begin{array}{l}
\alpha(X)=T_{g} \sin \left(\theta-\theta_{t}\right)+A \operatorname{sign}(\dot{\theta})+B(\dot{\theta})-\tau_{h}+\tau_{l} \\
\beta(X)=J \\
X=\left[\begin{array}{ll}
x_{1} & x_{2}
\end{array}\right]^{T}=[\theta \quad \dot{\theta}]^{T}
\end{array}\right.
$$

which leads to the following linearizing control input:

$$
\tau_{e}(t)=T_{g} \sin \left(\theta-\theta_{t}\right)+A \operatorname{sign}(\dot{\theta})+B(\dot{\theta})-\tau_{h}+\tau_{l}+J v
$$

Introducing the control input 8 (for sitting rehabilitation, with $\theta_{t}=\frac{\pi}{2}$ ) or 9 (for walking activity) into the dynamics (1) results in the following closed-loop (inner loop in the proposed framework of Fig. (1) linearized model:

$$
\left\{\begin{array}{l}
\dot{x}_{1}=x_{2} \\
\dot{x}_{2}=v \\
y=x_{1}
\end{array}\right.
$$

Because the expression of the position reference trajectory $\theta_{\text {ref }}$ is known, then the velocity and acceleration reference trajectories $\dot{\theta}_{\text {ref }}$ and $\ddot{\theta}_{\text {ref }}$, respectively can be easily computed using successive time derivatives. For the aim of stability analysis [19] of the resulting closed-loop dynamics introduced in section $\mathrm{V}$ hereafter, the definition of the following state errors are required:

$$
\begin{gathered}
\tilde{X}=X-X_{\text {ref }}=\left(\begin{array}{c}
\tilde{x}_{1} \\
\tilde{x}_{2}
\end{array}\right)=\left(\begin{array}{c}
\theta-\theta_{\text {ref }} \\
\dot{\theta}-\dot{\theta}_{\text {ref }}
\end{array}\right) \\
\dot{\tilde{x}}_{2}=\ddot{\theta}-\ddot{\theta}_{\text {ref }}=v-v_{\text {ref }}=\tilde{v} \text { with } v_{\text {ref }}=\ddot{\theta}_{\text {ref }}
\end{gathered}
$$

The obtained linear system (10) can now be discretized using Euler's method and a sampling period $T_{s}$, which leads to the following discrete state-space representation:

$$
\left\{\begin{array}{l}
\tilde{X}(k+1)=A \tilde{X}(k)+B \tilde{v}(k) \\
\tilde{y}(k)=C \tilde{x}(k)
\end{array}\right.
$$

with $A=\left(\begin{array}{cc}1 & T_{s} \\ 0 & 1\end{array}\right), B=\left(\begin{array}{c}0 \\ T_{s}\end{array}\right)$, and $C=\left(\begin{array}{ll}1 & 0\end{array}\right)$. Owing to the exact feedback linearization and the system discretization, the formulation of the MPC problem can now be easily defined, as introduced in the following section.

\section{PROPOSED MPC APPROACHES}

\section{A. A brief background on MPC}

MPC is a strategy widely adopted in the process industry and successfully implemented in various applications. It is an advanced control scheme based on the process model, which is used to predict the system future behavior over a certain prediction horizon, such that the best control action is selected [20]. A major advantage of MPC is its ability to implicitly consider constraints imposed on the system inputs and/or outputs by including them in the design process [21]-[22]. Its simple and intuitive design principle makes it a very popular choice in both the industry and in academia. The methodology of MPC is characterized by the following strategy: At each time instant $k$, the output of the system is measured. Based on this measurement, an optimal control sequence is computed. This sequence minimizes the cost function, which differs from one application to another, according to the user's expectations, over a finite horizon. Generally, it is assumed that the control 
signal varies over a control horizon with length $N_{c}$ (less than or equal to the length of the prediction horizon $N$, and remains constant over the interval $\left[N_{c}, N\right]$, with $N$ as the length of the prediction horizon. Only the first sample of this sequence is applied, and the same steps are repeated at time $k+1$ by shifting the prediction horizon. This update allows the consideration of uncertainties and/or disturbances that may occur while controlling the system [20]. The main limitation of this scheme is that, by considering the constraints imposed by the system, the MPC becomes relatively greedy in terms of computing time and memory space [23].

\section{B. Application of MPC to EICOSI orthosis}

Here, we propose the control of EICOSI orthosis using an MPC (block (ii) in the proposed framework of Fig. 1), to track the reference trajectory while smoothing the orthosis behavior. After identifying the system parameters, linearizing, and discretizing its dynamics, we can apply the proposed MPC approach to the studied lower-limb active orthosis as explained hereafter. The associated optimization problem can be expressed:

$$
\begin{aligned}
& V(k)=\min \left(\frac{1}{2} \tilde{v}_{\rightarrow k}^{T} H \tilde{v}_{\rightarrow k}+\tilde{X}(k)^{T} F \tilde{v}_{\rightarrow k}\right)+\frac{1}{2} \tilde{X}(k)^{T} Y \tilde{X}(k) \\
& \left\{\begin{array}{l}
\text { s.t } \theta_{\min } \leq \theta(k+i) \leq \theta_{\max } \text { for } i=1 . . N_{c} \\
\dot{\theta}_{\min } \leq \dot{\theta}(k+i) \leq \dot{\theta}_{\max } \text { for } i=1 . . N_{c} \\
\tilde{v}_{\min } \leq \tilde{v}(k+i) \leq \tilde{v}_{\max } \quad \text { for } i=1 . . N_{c} \\
\tilde{X}(0)=\tilde{X}(k) \\
\tilde{X}(k+i+1)=A \tilde{X}(k+i)+B \tilde{v}(k+i) \text { for } k \geq 0 \\
\tilde{y}(k+i)=C \tilde{X}(k+i) \text { for } k \geq 0
\end{array}\right.
\end{aligned}
$$

Iterating the state-space (13) over the prediction horizon gives:

$\left(\begin{array}{c}\tilde{X}(k+1) \\ \tilde{X}(k+2) \\ \vdots \\ \tilde{X}(k+N)\end{array}\right)=\left(\begin{array}{c}A \\ A^{2} \\ \vdots \\ A^{N}\end{array}\right) \tilde{X}(k)+\left(\begin{array}{cccc}B & 0 & \cdots & 0 \\ A B & B & \ddots & \vdots \\ \vdots & \ddots & \ddots & 0 \\ A^{N-1} B & \cdots & A B & B\end{array}\right)\left(\begin{array}{c}\tilde{v}(k) \\ \tilde{v}(k+1) \\ \vdots \\ \tilde{v}(k+N-1)\end{array}\right)$

which can be rewritten in a more compact form as:

$$
\tilde{X}_{\rightarrow k+1}=P_{X} \tilde{X}(k)+H_{X} \tilde{v}_{\rightarrow k}
$$

The first constraint in (14) is imposed by the mechanical stops of the exoskeleton. The second constraint is imposed by the physical limit of the actuator in terms of maximum speed. The angular speed of the knee joint is related to the motor angular speed. Accordingly, knowing the range of the motor, the equivalent range of the knee joint speed can be computed. Knowing that the reference trajectory is priori known and bounded in terms of $\theta_{r e f}, \dot{\theta}_{r e f}$, and $\ddot{\theta}_{r e f}$, the third constraint is discussed hereafter. Because the proposed control framework includes two control loops (an inner loop based on a nonlinear state feedback control law and an outer loop based on the proposed MPC/EMPC controller), we designed our EMPC controller considering the constraints on the control input $\tau_{e}$. In this study, the constraints on the linearized input have been computed based on those imposed on $\tau_{e}$. Using the relationship between these two variables expressed in (7), to maintain $\tau_{e}$ within its admissible values, we have to ensure that the right-hand side of this equation satisfies these bounds. By imposing constraints on the angular position and velocity, and because $\tau_{h}$ and $\tau_{l}$ are finite-valued torques, the constraints on the linearized input, which forces $\tau_{e}$ not to exceed its admissible values, can be deduced. Regarding the effect of the choice of these constraints on the cost function, it is noteworthy that altering each of these limits will change the size of the search space of the optimization problem. Accordingly, small limits will reduce the search space and may lead to suboptimal solutions, or even in extreme cases, to an unfeasible problem. However, dealing with an important number of varying parameters may cause discomfort to the wearer or a physical saturation. In summary, tuning is performed based on a compromise between the closed-loop desired performance and the physical limits of the system (in terms of joint position, speed, acceleration, and motor torque). In the proposed cost function $V(k) \sqrt{17}$, all the target performances are gathered and expressed mathematically. Specifically in this study, we attempt to reduce the tracking position error, as well as smoothen the EICOSI response by reducing the chattering phenomenon and the tracking velocity error. This approach was also selected because it helps to ensure guaranteeing an optimal trajectory tracking, even in the presence of parametric uncertainties originating from the identification process, and in the presence of external disturbances emerging from the interaction between the wearer and/or the ground. The weighting matrices $Q$ and $R$ in (17), are related to the state tracking errors and the acceleration, respectively while $Q_{f}$ is related to the terminal constraint. The cost function (17) was selected to meet our expectations in terms of tracking performance, as well as realize the closed-loop stability of the studied system. Initially, we set some conditions on the cost function tuning parameters (Please refer to Section $\mathrm{V}$ for more information), then we selected those that allow the desired performance to be achieved while satisfying these conditions:

$$
\begin{array}{r}
V(k)=\sum_{i=0}^{N-1}\left(\|\tilde{X}(k+i)\|_{Q}^{2}+\|\tilde{v}(k+i)\|_{R}^{2}\right) \\
+\tilde{X}(k+N)^{T} Q_{f}(N) \tilde{X}(k+N)
\end{array}
$$

This cost function can be represented in a compact form as:

$$
V(k)=\sum_{i=0}^{N-1} l(\tilde{X}(k+i), \tilde{v}(k+i))+F(\tilde{X}(k+N))
$$

By substituting $\tilde{X}(k+i)=A^{i} \tilde{X}(k)+\sum_{j=0}^{i-1} A^{j} B \tilde{v}_{k+i-1-j}$ into (18) and (14), we obtain the following equation :

$$
\left\{\begin{aligned}
& V(k)=\min \left(\frac{1}{2} \tilde{v}_{\rightarrow k}^{T} H \tilde{v}_{\rightarrow k}+\tilde{X}(k)^{T} F \tilde{v}_{\rightarrow k}\right) \\
&+\frac{1}{2} \tilde{X}(k)^{T} Y \tilde{X}(k) \\
& \text { s.t } G \tilde{v}_{\rightarrow k} \leq W+E \tilde{X}(k)
\end{aligned}\right.
$$

where $H, F, G, E, Y$ and $W$ are easily obtained from $Q, R, Q_{f}$ and (18) and (14). The constraints imposed by the system are omitted while calculating the MPC solution. This facilitates a significant reduction in computation time, and thus enables its real-time validation even with a small sampling period. In this case, the optimal control sequence is obtained by zeroing $\operatorname{grad}_{\tilde{v}_{\rightarrow k}} V(k)$. 


\section{Background on Explicit Model Predictive Control (EMPC)}

In the second part of this study, the constraints imposed by the system are considered while maintaining the same small sampling period. Even if efficient QP solvers based on interior point and active set methods exist, the computation of the control action $\tilde{v}(t)$ requires a significant online effort that makes MPC not applicable to fast systems with small sampling time, which is similar to the case of our study. Knowing that the sampling period is too small to enable the application of constrained MPC in real-time, we propose computing the possible control values offline using an EMPC method. This method is based on multi-parametric quadratic programming, which pre-solves the QP offline for the entire set of states. By considering $\bar{X}(t)$ as the vector of parameters, the EMPC moves the computation time offline by solving the optimization problem for all possible values of $\bar{X}(t)$ of interest, and making this dependence explicit. It converts the MPC into a continuous and piecewise-affine function of the parameter vector; thus enabling its application in realtime for fast systems. Generally, instead of computing the control online by minimizing the cost function, the problem is converted to a lookup table containing the optimal values of the gains that allow the MPC control law to be obtained explicitly as a function of the states, which considerably reduces the computation time [24].

\section{Application of EMPC to EICOSI orthosis}

Here, we consider the entire optimization problem as expressed in (19) (while considering the constraints imposed by the system). Let us consider $\bar{X}=\left(\begin{array}{lll}X & X_{\text {ref }} & \tau_{e}\end{array}\right)^{T}$ as the parameter vector of the problem in (19), which will be considered as a multi-parametric quadratic programming problem. Based on the Karush-Kuhn-Tucker (KKT) conditions for optimality, the authors of [25] proved that the optimizer function $\tilde{v}_{\rightarrow k}^{*}(x)$ is piecewise affine and continuous over the set of feasible parameters. They also verified that the corresponding optimal value $V(k)^{*}$ is continuous, convex, and piecewise quadratic. The algorithm of EMPC begins by selecting an arbitrary starting parameter vector $\bar{X}_{0}$ such as the origin. It then solves the QP problem to obtain the optimal solution $\tilde{v}\left(\bar{X}_{0}\right)^{*}$ and identify the subset of all active constraints represented by $\tilde{G} \tilde{v}(\tilde{X})=\tilde{S} \tilde{X}+\tilde{W}$, as well as the subset of inactive constraints represented by $\hat{G} \tilde{v}(\tilde{X}) \leq \hat{S} \tilde{X}+\hat{W}$.

\section{NDO AND REFERENCE TRAJECTORY GENERATOR}

\section{A. Application of NDO on the studied system}

In typical rehabilitation scenarios, when the wearer is asked to develop a muscle activation, the human torque $\tau_{h}$ is considered an external disturbance. In this case, we propose an NDO, the block $(i v)$ in the framework of Fig. 1, to estimate its values [26]. Accordingly, the dynamics (11) can be rewritten as follows:

$$
\dot{X}=F_{1}(X)+G_{1}(X) \tau_{e}+G_{2}(X) d
$$

where $G_{1}(X)=G_{2}(X)=\left(\begin{array}{c}0 \\ \frac{1}{J}\end{array}\right)$

During the sitting rehabilitation position, the human torque $\tau_{h}$ is considered an external disturbance and is estimated using the proposed NDO: $\hat{d}=\hat{\tau}_{h}$ and $F_{1}(X)$ is expressed as:

$$
F_{1}(X)=\left(\begin{array}{c}
\dot{\theta} \\
-\frac{1}{J}\left(-T_{g} \cos (\theta)+A \operatorname{sign}(\dot{\theta})+B(\dot{\theta})\right)
\end{array}\right)
$$

while for the standing position, we have two different cases related to the gait cycle phases. The torque $\tau_{l}$ differs between these two cases, as mentioned in Section II . During the swing phase, the lumped torque $\tau_{l}$ is represented by (2); whereas, in the stance phase, it is considered an external disturbance, and can be estimated with the human torque using the NDO. During the swing phase, the human torque $\tau_{h}$ is considered as an external disturbance and is estimated using the NDO: $\hat{d}=\hat{\tau}_{h}$ and

$$
F_{1}(X)=\left(\begin{array}{c}
\dot{\theta} \\
-\frac{1}{J}\left(T_{g} \sin \left(\theta-\theta_{t}\right)+A \operatorname{sign}(\dot{\theta})+B(\dot{\theta})+\tau_{l}\right)
\end{array}\right)
$$

while during the stance phase, the human $\tau_{h}$ and lumped $\tau_{l}$ torques are both estimated using the NDO: $\hat{d}=\hat{\tau}_{h}+\hat{\tau}_{l}$ and

$$
F_{1}(X)=\left(\begin{array}{c}
\dot{\theta} \\
-\frac{1}{J}\left(T_{g} \sin \left(\theta-\theta_{t}\right)+A \operatorname{sign}(\dot{\theta})+B(\dot{\theta})\right)
\end{array}\right)
$$

The proposed nonlinear observer is designed:

$$
\left\{\begin{array}{l}
\hat{d}=z+p(X) \\
\dot{z}=L\left(-F_{1}(X)-G_{1}(X) \tau_{e}-G_{2}(X)(z+p(X))\right)
\end{array}\right.
$$

where $\hat{d}=\hat{\tau}_{h}, p(X)=k_{1} \theta+k_{2} \dot{\theta}, L=\frac{\partial p(X)}{\partial X}=\left(\begin{array}{ll}k_{1} & k_{2}\end{array}\right)$, where $k_{1}$ and $k_{2}$ are two positive constants that allows us to deduce the unknown human/human-lumped torque values (considered here as an external disturbance) without using an additional sensor, thus reducing both the cost and dimensions of the studied active orthosis.

\section{B. Reference trajectories generator}

In this study, we adopt a time-based algorithm that facilitates the generations of a real-time knee joint reference trajectory by interpolating a number of samples matched with each phase sample speed (e.g., before stance/swing length) using a cubic spline function. The pre-allocated samples are extracted from the gait profile analyses of 20 healthy subjects [27]. The resulting generated trajectory is illustrated in Fig 3

\section{Closed-Loop Stability ANALYSis}

To validate the asymptotic stability of the resulting closedloop system, we need to demonstrate, via the direct method presented in [19], that the cost function (18) is decreasing. Verifying that the objective function is a Lyapunov candidate ensures that this function decreases; hence, the controlled system is stable:

$$
\left\{\begin{array}{l}
V(k+1)-V(k)=\Delta V(k)<0 \text { for }(\tilde{X}, \tilde{v}) \neq(0,0) \\
\Delta V(k)=0 \text { if }(\tilde{X}, \tilde{v})=(0,0)
\end{array}\right.
$$

In [19], the closed-loop asymptotic stability of an MPC system is validated by satisfying:

$$
V(k+1)-V(k)+l(\tilde{X}(k), \tilde{v}(k)) \leq 0
$$


and the following assumptions A1-A4:

A1: $X_{f} \subset \mathbb{X}, X_{f}$ closed, $0 \in X_{f}$ (state constraint satisfied in $X_{f}$ ).

A2 : $\quad K_{f}(x) \in \mathbb{U}, \forall x \in X_{f}$ (control constraint satisfied in $\left.X_{f}\right)$.

A3 : $f\left(x, K_{f}(x)\right) \in X_{f}, \forall x \in X_{f}\left(X_{f}\right.$ is a positively invariant under $\left.K_{f}().\right)$.

A4 : $[F+\ell]\left(x, K_{f}(x)\right) \leq 0, \forall x \in X_{f}(F($.$) is a local$ Lyapunov function).

By designing the cost function as expressed in (17)-(18), we can easily verify that:

$$
l(\tilde{X}(k+i), \tilde{v}(k+i))=\|\tilde{X}(k+i)\|_{Q}^{2}+\|\tilde{v}(k+i)\|_{R}^{2}
$$

is a Lyapunov function candidate because

$$
\left\{\begin{array}{l}
l(\tilde{X}(k+i), \tilde{v}(k+i))>0 \text { for }(\tilde{X}, \tilde{v}) \neq(0,0) \\
l(0,0)=0
\end{array}\right.
$$

In this case, inequalities (25) and (26) become equivalent because $l(\tilde{X}(k), \tilde{v}(k))$ is a Lyapunov function. The cost function at the time step $k+1$ is equal to:

$$
\begin{aligned}
V(k+1)= & \sum_{i=0}^{N-1}\left(\|\tilde{X}(k+i+1)\|_{Q}^{2}+\|\tilde{v}(k+i+1)\|_{R}^{2}\right) \\
& +\tilde{X}(k+N+1)^{T} Q_{f}(N+1) \tilde{X}(k+N+1)
\end{aligned}
$$

A simple mathematical reformulation gives the expression:

$$
\begin{array}{r}
V(k+1)=\sum_{i=1}^{N}\left(\|\tilde{X}(k+i)\|_{Q}^{2}+\|\tilde{v}(k+i)\|_{R}^{2}\right) \\
+\tilde{X}(k+N+1)^{T} Q_{f}(N+1) \tilde{X}(k+N+1)
\end{array}
$$

In the following, we express $V(k+1)$ as a function of $V(k)$ to simplify the computation of $\Delta V(k)$ :

$$
\begin{gathered}
V(k+1)=V(k)+\tilde{X}(k+N)^{T} Q(N) \tilde{X}(k+N)-l(\tilde{X}(k), \tilde{v}(k)) \\
+R(N) \tilde{v}(k+N)^{2}-\tilde{X}(k+N)^{T} Q_{f}(N) \tilde{X}(k+N) \\
+\tilde{X}(k+N+1)^{T} Q_{f}(N+1) \tilde{X}(k+N+1) .
\end{gathered}
$$

Because $\tilde{X}(k+N+1)=A \tilde{X}(k+N)+B \tilde{v}(k+N)$, then:

$$
\begin{gathered}
V(k+1)-V(k)+l(\tilde{X}(k), \tilde{v}(k))=\tilde{X}(k+N)^{T}(Q(N) \\
\left.-Q_{f}(N)+A^{T} Q_{f}(N+1) A\right) \tilde{X}(k+N) \\
+2 \tilde{X}(k+N)^{T} A^{T} Q_{f}(N+1) B \tilde{v}(k+N) \\
+\left(R(N)+B^{T} Q_{f}(N+1) B\right) \tilde{v}(k+N)^{2}
\end{gathered}
$$

By choosing $l(\tilde{X}, \tilde{v})$ as a Lyapunov function $(l(0,0)=0$ and $l(\tilde{X}, \tilde{v})>0, \forall(\tilde{X}, \tilde{v}) \neq(0,0))$, if we compute 32 and demonstrate that its right hand side is negative, we can then prove that the cost function is a Lyapunov candidate and consequently deduce the asymptotic stability of the closedloop system. Accordingly:

$$
\begin{aligned}
& V(k+1)-V(k)+l(\tilde{X}(k), \tilde{v}(k)) \leq 0 \\
& \Rightarrow \Delta V(k+1) \leq-l(\tilde{X}(k), \tilde{v}(k))<0
\end{aligned}
$$

Equation $\sqrt{33}$ is verified for all $(\tilde{X}, \tilde{v}) \neq(0,0)$ because $l(\tilde{X}, \tilde{V})$ is a Lyapunov function. This can be satisfied if the tuning parameters $Q, Q_{f}, R, N$, and $N_{c}$ are carefully selected. To ensure that, we impose a few conditions and summarize them in (34). If these conditions are satisfied, the negativity of the right hand side of (32) is guaranteed; thus the asymptotic stability of the controlled system is proved. This is expressed in the following equation as:

$$
\left\{\begin{array}{l}
Q(N)-Q_{f}(N)+A^{T} Q_{f}(N+1) A \leq 0 \\
R(N)+B^{T} Q_{f}(N+1) B \leq 0 \\
\tilde{X}(k+N)^{T} A^{T} Q_{f}(N+1) B \tilde{v}(k+N) \leq 0 \\
V(k) \geq 0, \quad \forall k \geq 0
\end{array}\right.
$$

We choose the weighting matrices $Q$ and $Q_{f}$ as follows:

$$
=\left(\begin{array}{cccc}
\left(\begin{array}{cc}
q^{2} & 0 \\
0 & m^{2}
\end{array}\right) & \left(\begin{array}{cc}
0 & 0 \\
0 & 0
\end{array}\right) & \cdots & \left(\begin{array}{cc}
0 & 0 \\
0 & 0
\end{array}\right) \\
\left(\begin{array}{cc}
0 & 0 \\
0 & 0
\end{array}\right) & \left(\begin{array}{cc}
q^{3} & 0 \\
0 & m^{3}
\end{array}\right) & \ddots & \vdots \\
\vdots & & \ddots & \vdots \\
\vdots & \ddots & \left(\begin{array}{cc}
q^{N} & 0 \\
0 & m^{N}
\end{array}\right) & \left(\begin{array}{cc}
0 & 0 \\
0 & 0
\end{array}\right) \\
\left(\begin{array}{ll}
0 & 0 \\
0 & 0
\end{array}\right) & \cdots & \left(\begin{array}{cc}
0 & 0 \\
0 & 0
\end{array}\right) & \left(\begin{array}{cc}
q & 0 \\
0 & m
\end{array}\right)
\end{array}\right)
$$

Therefore, the weighting matrix applied to the $N^{t h}$ term of the cost function is equal to $Q(N)=\left(\begin{array}{cc}q & 0 \\ 0 & m\end{array}\right)$. In addition, we can select the terminal-weighting matrix as $Q_{f}(N+i)=\left(\begin{array}{cc}-(N+i)(q+1) & 0 \\ 0 & -N m(i+1)^{N}\end{array}\right)$.

In this case, we obtain:

$$
M(N)=Q(N)-Q_{f}(N)+A^{T} Q_{f}(N+1) A
$$

which can be represented as $M(N)=\left(\begin{array}{ll}a & b \\ c & d\end{array}\right)$, where $a=$ $-1, b=c=-T_{s}(N+1)(q+1), d=(N+1)\left(m-T_{s}^{2}(q+\right.$ 1)) $-2^{N} N m$. To ensure that $M(N)$ is a negative semidefinite matrix, its principle minors must satisfy the conditions $\Delta_{1}=$ $\operatorname{det}(a) \leq 0$ and $\Delta_{2}=\operatorname{det}(M) \geq 0$. Their computation leads to $\Delta_{1}=-1$, then the first condition is always satisfied and $\Delta_{2}=-m(N+1)+T^{2}(N+1)(q+1)+2^{N} N m-T^{2}(N+1)^{2}(q+1)^{2}$. To satisfy the latter condition, we choose $m$ as follows:

$$
m \geq \frac{T^{2}(N+1)(q+1)(N q+N+q)}{N\left(2^{N}-1\right)-1}
$$

To ensure that $R(N)+B^{T} Q_{f}(N+1) B \leq 0$, the following condition should be satisfied:

$$
R(N) \leq 2^{N} N T^{2} m
$$

Let us now consider the third condition in (34). The state-space equation at time step $k+N$ is equal to:

$$
\tilde{X}(k+N)=A \tilde{X}(k+N-1)+B \tilde{v}(k+N-1)
$$

Computing (38) with $A$ and $B$ as the respective state and input matrices, defined in (13), leads to:

$$
\tilde{x}_{2}(k+N)=\tilde{x}_{2}(k+N-1)+T_{s} \tilde{v}(k+N-1)
$$

For a control horizon satisfying $N_{c} \leq N-1$, we can write:

$$
\tilde{x}_{2}(k+N)=\tilde{x}_{2}(k+N-1)+T_{s} \tilde{v}(k+N)
$$


because $\tilde{v}(k+i)=\tilde{v}\left(N_{c}\right), \forall i \geq N_{c}$, then

$$
\tilde{v}(k+N)=\tilde{v}(k+N-1)) .
$$

Using the Z-transform, we obtain:

$$
\tilde{x}_{2}(z)=T_{s} \frac{z}{z-1} \tilde{v}(z)
$$

which is equivalent to:

$$
\tilde{x}_{2}(k+N)=T_{s} \Gamma(k+N) \tilde{v}(k+N)
$$

with $\Gamma(k+N)$ being the unit step function. This proves that $\tilde{x}(k+N)$ and $\tilde{v}(k+N)$ have the same sign; hence, the following inequality is always satisfied:

$$
\begin{aligned}
& \tilde{X}(k+N)^{T} A^{T} Q_{f}(N+1) B \tilde{v}(k+N) \\
& \quad=-2^{N} T_{s} N m \tilde{x}_{2}(k+N) \tilde{v}(k+N) \leq 0
\end{aligned}
$$

With $Q$ and $Q_{f}$ equal to those chosen in this study, and the tuning parameters $q, m, N$, and $R(N)$ satisfying the following conditions:

$$
\left\{\begin{array}{l}
m \geq \frac{T_{s}^{2}(N+1)(q+1)(N q+N+q)}{N\left(2^{N}-1\right)-1} \\
R(N) \leq 2^{N} N T_{s}^{2} m \\
R(N), q, m, N \geq 0 \\
0 \leq N_{c} \leq N-1
\end{array}\right.
$$

this proves that 33 is satisfied for all $k \geq 0$ and $(\tilde{X}, \tilde{v}) \neq$ $(0,0)$. For $(\tilde{X}, \tilde{v})=(0,0)$, the cost function is equal to:

$$
V(k)=\sum_{i=0}^{N-1} l(0,0)+\tilde{X}(k+N)^{T} Q_{f}(N) \tilde{X}(k+N)=0
$$

Accordingly, $l(0,0)=0$ and $\tilde{X}(k+N)=0$ because $(\tilde{X}, \tilde{v})=$ $(0,0)$. This proves that the cost function $V(k)$ is a Lyapunov candidate because the following conditions are satisfied for all $k \geq 0$ :

$$
\left\{\begin{array}{l}
\Delta V(k)<0 \forall(\tilde{X}, \tilde{v}) \neq(0,0) \\
V(k)=0 \text { for }(\tilde{X}, \tilde{v})=(0,0)
\end{array}\right.
$$

This concludes the asymptotic stability of the resulting closedloop system. The proposed EMPC controller inherits both the stability and performance properties of the MPC [25].

\section{EXPERIMENTAL Methods}

In this section, we give a brief overview of the experimental operating conditions, the performed experimental scenarios, and the proposed metrics used to assess the performances of the proposed controllers.

Our experiments were conducted with three healthy subjects (gender: 3 males; age: $30 \pm 3$ years old; height: $1.72 \pm 0.04$ $\mathrm{m}$; weight: $67 \pm 8 \mathrm{~kg}$ ) while wearing the exoskeleton (cf. TABLE [). The wearers, who were explicitly informed about the experimental protocol and the expectations of this study, gave their consent before participating in the experiments. All subjects can ensure a complete flexion and extension of the knee joint without spasticity or contracture. Constraints on the knee joint position and velocity of the orthosis between the full knee extension $\left(0^{\circ}\right)$ and flexion $\left(135^{\circ}\right)$, are guaranteed by adopting mechanical stops. All precautions were taken to protect the health of the subjects who served as research subjects. Precautions were also taken to protect the privacy of the research subjects and their confidentiality. In section VII], we attempt to validate the proposed MPC-based control framework on the subjects wearing the active orthosis in three rehabilitation scenarios (i) in a sitting position where the hip angle is constant without ground interaction, and while walking on a treadmill with (ii) normal, and (iii) abnormal, walking gaits, respectively. In the last two scenarios, the interaction with the ground is considered as an external perturbation, and the hip angle is no longer constant. The proposed NDO is used to estimate the exerted human/human-lumped torque. To assess the performances of the proposed controllers, the following metrics have been analyzed: (i) the EMG activation of the muscles during walking and (ii) the exoskeleton torque required by the AAFO to provide functional assistance and satisfactory tracking of the desired trajectory. Indeed, exoskeleton torque, human torque and muscle activation, are good indicators of the subject involvement in the rehabilitation process. The EMG signals were sampled at $100 \mathrm{~Hz}$. The raw EMG signals were filtered using a high-pass fourth order Butterworth filter with a cutoff frequency at $30 \mathrm{~Hz}$, full wave rectified, and then filtered using a low-pass fourth order Butterworth filter with a cutoff frequency at $2 \mathrm{~Hz}$ [28] Figs. 4, 5, and 7 illustrate the evolution of the angular position and velocity, estimated human torque, and measured exoskeleton torque. Processed muscular activities of both Vastus Medialis (VM) and Biceps Femoris (BF) are also illustrated. The reference trajectories representing the walking profile of a healthy subject are depicted in red while the measured ones are in black for S1 and S2 and in green for S3. Each scenario was repeated three times with the concerned subject, and each time a different controller is applied (PID / MPC and EMPC). It should be mentioned that the wearers were only informed about the experimental protocol and the expectations of the study, without any indication on which controller was running in each session to not alter their performances and induce any bias in their movements. For each subject, the controller order was chosen randomly and only the operator was aware about which controller was running. The participants had no previous experiences with the exoskeleton, they were simply asked to perform some training movements with the robot, in both sitting and walking conditions prior to the experiment sessions, to get used to the robot and ensure they can perform movements comfortably. Each experimental session lasted for 60-s per controller/subject of a treadmill walking (60-s for the PID, 60-s for MPC, 60-s for the EMPC) while wearing the exoskeleton. After each session, participants were asked to sit on a chair during 3 minutes for rest. To compare the performance of each controller at the same scale and for the sake of clarity, we have extracted some of the obtained results with each controller (PID,MPC and EMPC) and have concatenated them together in Figs. 4, 5 and 7 . During the swing phase, the lumped torque is computed using (2), while it is considered as an external disturbance and is estimated with the human torque via the NDO during the stance phase. 


\section{RESULTS AND DISCUSSIONS}

In this section, we present and discuss the obtained results of the conducted experimental study to evaluate the performances of the proposed control framework with MPC and EMPC in three different scenarios. The performances of the proposed controllers are compared with a tuned PID controller, based on trial-and-error, until it reaches its best performances in terms of settling time, precision tracking, and stability. Today, PID controllers are used in most automatic process control applications in the industry to regulate flow, temperature, pressure, position, speed, level, and several other industrial process variables. In wearable robotics, beyond academia, PID-based controllers have also been adopted in several commercialized exoskeletons.

\section{A. Scenario 1: Sitting position with two rehabilitation modes}

In this first case, we ask subject $\mathrm{S} 1$ to wear the exoskeleton and to perform a movement of flexion/extension while sitting, without any interaction with the ground. This 20-s rehabilitation exercise is divided into two different parts. During the first 10-s, the wearer is asked to provide an assistive force by following the reference trajectory displayed on the laptop screen in real-time. During the last 10 -s, he is asked to resist towards the desired trajectory by providing a resistive effort in the opposite direction. All the generated desired trajectories are presented in real-time on a laptop screen in front of the wearer while the change between the different phases is indicated by a beep. Only one gait cycle data per assistive/resistive phase and per controller (PID/MPC/EMPC) was cropped and all of them were concatenated together in Fig 4 This Figure clearly demonstrates that both MPC and EMPC exhibit better performances than PID controller in terms of position and velocity tracking. It can be noted that during the assistive scenario (first 10-s), the three controllers succeeded in tracking the desired trajectory. When comparing the results of the three controllers with respect to the resistive scenario, it can be demonstrated that both MPC and EMPC exhibited better tracking performances than PID while preserving a relatively smooth motion. The PID controller was unable to reach the peak values of the reference trajectory and exhibited a poor tracking performance.

\section{B. Scenario 2: Healthy walking pattern}

In this scenario, subject $\mathrm{S} 1$ is asked to walk on a treadmill for 30-s while wearing the exoskeleton. The reference trajectory is generated via a fixed-time based method, as described in Section IV-B Fig 5 gathers the obtained results with the three used controllers, where only 10-s of each controller performance are cropped and concatenated together. This figure indicates that MPC and EMPC ensure better position and velocity tracking performances than the PID controller. Because the NDO-estimated torque includes $\tau_{h}$ and $\tau_{l}$ together, it would be more reliable to use the EMG signals to interpret the results of the walking experiments. The EMG curves of both BF and VM muscles exhibit higher values when PID controller is used, which indicates the superiority of the

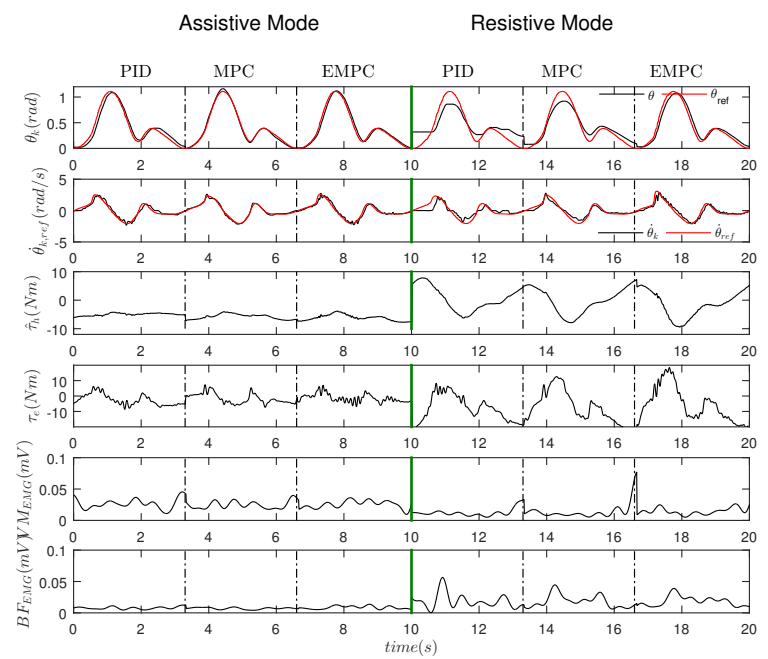

Fig. 4: S1 - Using PID, MPC, and EMPC controllers in a sitting position while exerting an assistive effort during the first 10-s and a resistive effort during the last 10 -s.

MPC based controllers in providing better assistance than the PID controller, during the walking activity.

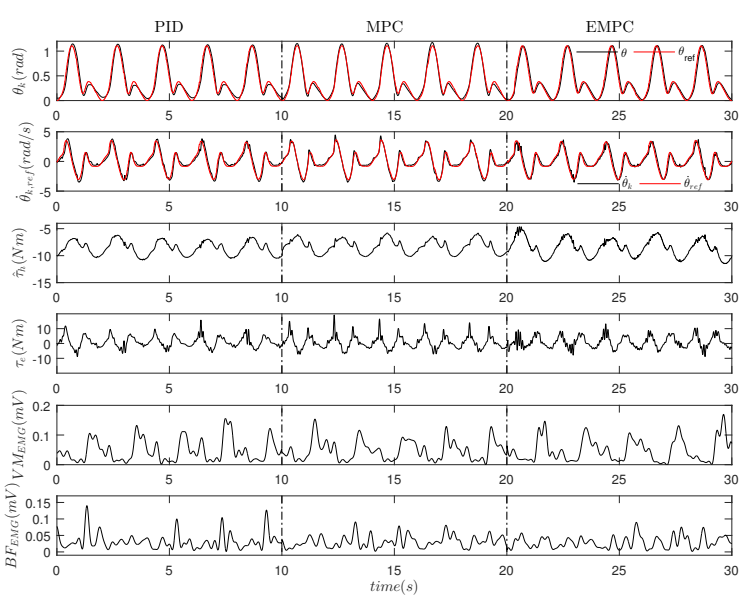

Fig. 5: S1 - Walking with a normal pattern while using PID [0,10], MPC $[10,20]$ and EMPC $[20,30]$ controllers.

\section{Scenario 3: Abnormal walking pattern}

Because the walking gaits of people with reduced mobility differ from those of healthy people (as shown in Fig. 6, and to validate the robustness of the proposed approaches, two healthy subjects (S2 and S3) were asked to mimic the behavior of people suffering from lower-limb muscular weaknesses. These subjects did not participate in the previous set of experiments with a normal gait. S2 and S3 were trained to mimic abnormal gaits while wearing the active orthosis and walking on a treadmill, with a pre-selected speed of $2 \mathrm{~km} / \mathrm{h}$ for 60 -s, under three different assistive scenarios. Each experimental test had a duration of $60 \mathrm{~s}$, where the useful data were extracted from the last $30 \mathrm{~s}$, while the first $30 \mathrm{~s}$ were used to enable the wearer to adapt his walking pattern to the abnormal gait. Indeed, $10 \mathrm{~s}$ of each test (PID, MPC, and EMPC) were cropped and concatenated together 
in Fig. 7 for the two subjects to enable the comparison of the controllers. It can be deduced from Figs. 7 and 8 that in terms of position and velocity knee joint tracking, both MPC and EMPC exhibit better results than the conventional PID controller. In fact, a relatively important tracking error can be observed in PID controller case, in particular, during the stance phase, owing to the ground reaction force acting as external perturbation. This error was reduced when using the MPC. In the case of the EMPC scheme, the NDO compensates for this disturbance in the same time step. Similarly, the error is reduced during the swing phase while using both MPC and EMPC. Furthermore, the MPC exhibits less control effort and better tracking performances than the PID. In addition, the EMPC required further control torque to ensure better tracking performances than both the PID and MPC controllers.
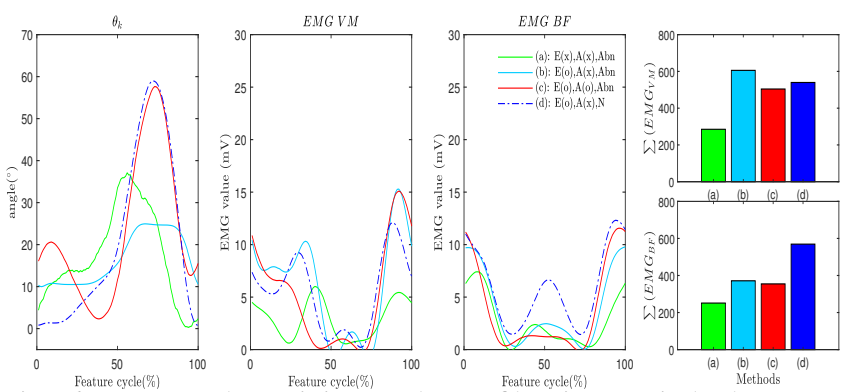

Fig. 6: Average knee joint angle profiles in saggital plane, EMG profiles of the vastus medialis (VM) and biceps femoris (BF) along $100 \%$ gait cycles for different conditions. (a): the subject walks with abnormal gait without wearing the knee joint orthosis, (b): the subject walks with abnormal gait while wearing the orthosis but without torque assistance, (c): the subject walks with abnormal gait with the torque assistance from the orthosis using proposed control method, and (d): the subject walks with normal gait while wearing the orthosis but without torque assistance. The right bar plot shows the VM and BF EMG activities in the different cases by using trapezoidal numerical integration with respect to 100 cycle points.

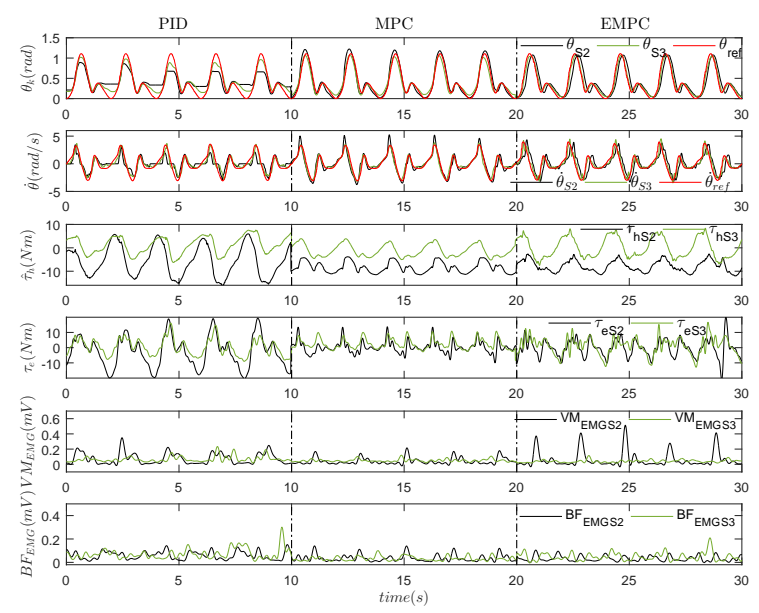

Fig. 7: S2/S3 - Walking with an abnormal pattern while using PID [0,10], MPC [10,20] and EMPC [20,30] controllers.

It is worth noting that the EMG recordings exhibit less activation in the MPC case than the PID and EMPC controllers. In fact, the VM muscular group exhibits an important activation in the PID and EMPC cases to compensate the body weight
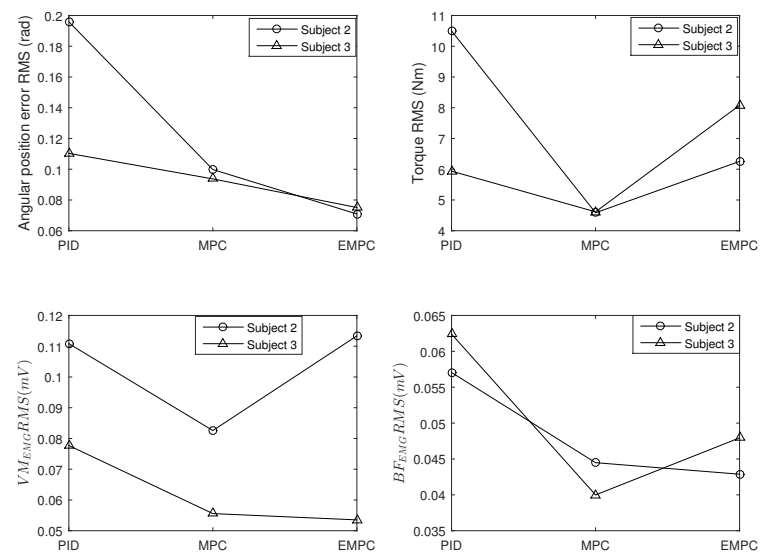

Fig. 8: S2/S3 - Root mean square (RMS) errors for angular position, torque, $V M_{E M G}$ and $B F_{E M G}$, versus controllers.

during the heel strike of the stance phase. Furthermore, during late swing phase, the BF muscular group exhibits an important activation to ensure sufficient clearance of the swinging leg following the knee joint flexion. For both subjects, the EMG results for MPC exhibit relatively reduced peak activation, as illustrated in Fig 7. In summary, according to the proposed metrics and the obtained results, in general, the proposed predictive controllers (MPC and EMPC) outperform the PID controller. Furthermore, although EMPC requires a larger control input than the conventional MPC, it exhibits the best performances among the controllers, in terms of trajectory tracking for all subjects.

The obtained experimental results of scenario 3 are illustrated in the video available at: https ://youtu.be/tfvGgg4aFm0

\section{CONCLUSION AND FUTURE WORK}

In this study, we propose a novel MPC framework dedicated to assistive and rehabilitation purposes, by adopting an actuated knee joint orthosis. The proposed framework includes $(i)$ an exact input-to-state feedback linearization, (ii) a dedicated MPC or EMPC, (iii) a least-squares dynamic parameters identification, (iv) an NDO, (v) a Lyapunov-based stability analysis, and (vi) a fixed time based trajectory generator. Because considering the imposed constraints makes the MPC controller consume more computing time and memory space, these constraints are omitted in the first proposed controller (MPC). To address this limitation, an explicit MPC (EMPC) scheme is proposed, which can consider the system constraints. The proposed framework was validated via realtime experiments performed on three healthy subjects wearing the active orthosis while sitting, and while walking on a treadmill with normal and abnormal gait walking patterns. The performances of the proposed controllers are compared with those of a conventional PID controller. The obtained results clearly indicate that the proposed predictive controllers outperform the PID controller in terms of tracking performances, orthosis generated torque, and EMG activities of the involved muscles. Our future work will focus on realtime MPC control implementation with larger population of 
subjects including real patients, while considering the effects of (i) model uncertainties, (ii) external disturbances, and (iii) noises on the control performances and stability, and reducing the conservative behavior of MPC.

\section{REFERENCES}

[1] World Health Organization: "Ageing and health", [Online]. Available: http://www.who.int/news-room/fact-sheets/detail/ageing-and-health. , Feb 5. 2018

[2] B. Chen, H. Ma, L.Y Qin, F. Gao, K.M Chan, S.W Law, L. Qin and W.H Liao, "Recent developments and challenges of lower extremity exoskeletons", Journal of Orthopedic Translation, vol. 5, pp. 26-37, Dec. 2016.

[3] S. Mohammed and Y. Amirat, "Towards Intelligent Lower Limb Wearable Robots: Challenges and Perspectives - State of the Art", IEEE International Conference on Robotics and Biomimetics, Feb. 2009.

[4] K. I. K. Sherwani, N. Kumar, A. Chemori, M. Khan, S. Mohammed, "RISE-based adaptive control for EICoSI exoskeleton to assist knee joint mobility", Robotics and Autonomous Systems, vol. 124, 2020

[5] S. Mohammed, Y. Amirat, H. Rifai, "Lower-Limb Movement Assistance through Wearable Robots: State of the Art and Challenges", Advanced Robotics., vol. 26, pp. 1-22, 2012.

[6] B. Chen, B. Zi, Z Wang, L. Qin , W. H. Liao, "Knee exoskeletons for gait rehabilitation and human performance augmentation: A state-of-theart" , Mechanism and Machine Theory, Elsevier, vol. 134, pp. 499511, Jan. 2019.

[7] M. K. Shepherd, E. J. Rouse, "Design and Validation of a TorqueControllable Knee Exoskeleton for Sit-to-Stand Assistance", IEEE/ASME Transactions on Mechatronics, 2016.

[8] W. Huo, S. Mohammed, Y. Amirat, and K. Kong, "Active Impedance Control of a Lower Limb Exoskeleton to Assist Sit-to-Stand Movement", IEEE International Conference on Robotics and Automation, 2016.

[9] K Junius, B. Brackx, V. Grosu, H. Cuypers, J. Geeroms, M. Moltedo, B. Vanderborght, D. Lefeber, "Mechatronic Design of a Sit-to-Stance Exoskeleton", IEEE RAS and EMBS International Conference on Biomedical Robotics and Biomechatronics, 2014.

[10] K. Kamalia, A. A. Akbaria, A. Akbarzadeh, "Trajectory generation and control of a knee exoskeleton based on dynamic movement primitives for sit-to-stand assistance", Advanced Robotics, 2016.

[11] J. Wang, X. Li, T. Huang, S. Yu, Y. Li, T. Chen, A. Carriero, M. Oh-Park, H. Su, "Comfort-Centered Design of a Lightweight and Backdrivable Knee Exoskeleton", IEEE Robotics and Automation Letters, 2018.

[12] H. Zhu, C. Nesler, N. Divekar, M. T. Ahmad, R. D. Gregg, "Design and Validation of a Partial-Assist Knee Orthosis with Compact, Backdrivable Actuation", IEEE 16th International Conference on Rehabilitation Robotics, 2019

[13] G. Lv, H. Zhu, R. D. Gregg, "On the Design and Control of Highly Backdrivable Lower-Limb Exoskeletons", IEEE Control Systems Magazine, 2018.

[14] S. Mohammed, H. Rifai, B. Daachi and Y. Amirat, "Adaptive Control of a Human-Driven Knee Joint Orthosis", IEEE International Conference on Robotics and Automation, pp. 2486-2491, 2012

[15] H. Rifai, M-S. Ben Abdessalem, A. Chemori, S. Mohammed and Y. Amirat, "Augmented L1 Adaptive Control of an Actuated Knee Joint Exoskeleton: From Design to Real-Time Experiments", IEEE ICRA16, Stockholm, Sweden, 2016.

[16] S. Mohammed, H. Rifa, W. Hassani and Y. Amirat, "Nested saturation based control of an actuated knee joint orthosis", Mechatronics, vol. 23, no. 8, pp. 1141-1149, Sep. 2013.

[17] W. Huo, S. Mohammed, Y. Amirat, "Impedance Reduction Control of a Knee Joint Human-Exoskeleton System", IEEE Transactions on Control Systems Technology, 2018

[18] R. Krneta, S. Antic, and D. Stojanovic, "Recursive Least Squares Method in Parameters Identification of DC Motors Models", Ser: Elec. Energ, vol. 18, no. 3, pp. 467-478, Dec. 2005.

[19] D. Q. Mayne, J. B. Rawlings, C. V. Rao, P. O. M. Scokaert, "Constrained model predictive control: Stability and optimality", Automatica, vol. 36 no. 6, pp. 789-814, 2000.

[20] M. Yue, C. An and J.Z. Sun, "An Efficient Model Predictive Control for Trajectory Tracking of Wheeled Inverted Pendulum Vehicles with Various Physical Constraints", International Journal of Control, Automation and Systems., pp. 265-274, 2018.

[21] J. Kohler, M. A. Muller, F. Allgower, "A nonlinear model predictive control framework using reference generic terminal ingredients", IEEE Transactions on Automatic Control, vol. 65, issue 8, pp. 3576-3583, 2020.
[22] E. F. Camacho and C. Bordons, "Model Predictive Control", Ed. Springer (2nd Edition)., London, UK, 2007.

[23] X. Li, L. Han, H. Liu, W. Wang and C. Xiang, "Real-time Optimal Energy Management Strategy for a Dual-Mode Power-Split Hybrid Electric Vehicle Based on an Explicit Model Predictive Control Algorithm", Energy., 2019

[24] A. Bemporad, M. Morari, V. Dua, E. N. Pistikopoulos, "The explicit linear quadratic regulator for constrained systems", Automatica., vol. 37 , pp. 3-20, 2002.

[25] G. Bardaro, L. Bascetta, E. Ceravolo, M. Farina, M. Gabellone and M. Matteuccir, "MPC-based control architecture of an autonomous wheelchair for indoor environments", Control Engineering Practice, vol. 78, pp. 160-174, 2018.

[26] S. Mohammed, W. Huo, J. Huang, H. Rifai, Y. Amirat, "Nonlinear Disturbance Observer Based Sliding Mode Control of a Human-Driven Knee Joint Orthosis", Robotics and Autonomous Systems, 2014.

[27] E. Hutin, D. Pradon, F. Barbier, B. Bussel, J.M Gracies, N. Roche, " Walking velocity and lower limb coordination in hemiparesis", Gait and Posture, 2012.

[28] W. Hassani, S. Mohammed, H. Rifai, Y. Amirat, EMG Based Approach for Wearer-centered Control of a Knee Joint Actuated Orthosis, IEEE/RSJ International Conference on Intelligent Robots and Systems (IROS), Japan, 2013.

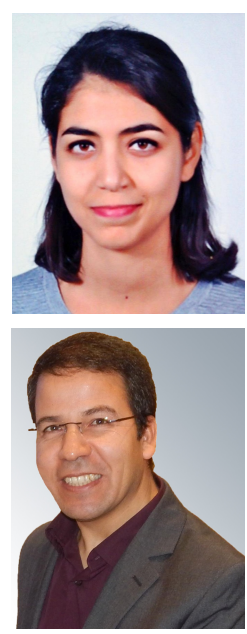

Ines Jammeli is a Ph.D. student at ENICarthage University in Tunisia. She is currently pursuing her Ph.D. in the research Laboratory Laboratory of Advanced Systems at the Tunisia Polytechnic High School-University of Carthage under the supervision of Salwa Elloumi, Ahmed Chemori and Samer Mohamed. She received her Mechatronics engineering degree at the University of ENICarthage in 2016.

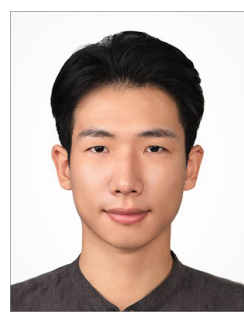

Ahmed Chemori received the M.Sc. and Ph.D. degrees both in automatic control from the Grenoble Institute of Technology, Grenoble, France, in 2001 and 2005, respectively. He has been a Postdoctoral Fellow with the Automatic Control Laboratory, Grenoble, France, in 2006. He is currently a tenured Research Scientist in automatic control and robotics with LIRMM laboratory. His research interests include nonlinear, adaptive, and predictive control and their applications in robotics.

Huiseok Moon received the B.S. degree in division of robotics from Kwangwoon University, Seoul, South Korea in 2017, and the M.S. degree in mechanical engineering from Sogang University, Seoul, South Korea in 2019, respectively. In 2019, he joined the University Paris-Est Crteil - UPEC, France, where he is currently a candidate of Ph.D. His current research interests include modelling and control of human-robot physical interaction systems also known as wearable robots.

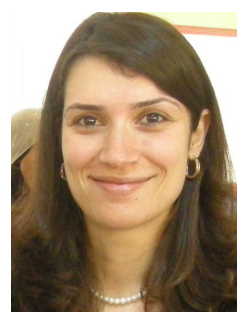

Salwa Elloumi received the master degree of $\mathrm{Au}$ tomatic control in 2000, the $\mathrm{PhD}$ degree in 2005 and the University habilitation (HDR) in 2014 in Electrical Engineering, all from the National High School of Engineers of Tunis. She is currently Professor in National Engineering School of Carthage and member of the research Laboratory of Advanced Systems at Polytechnic High School of Tunisia. Her current research interests include control of complex systems.

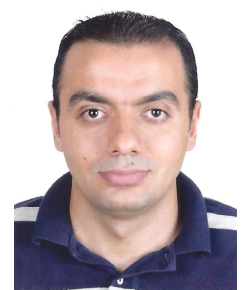

Samer Mohammed received the Ph.D. degree from the University of Montpellier (LIRMM/CNRS), Montpellier, France, in 2006. He is currently a Professor with the Laboratory of Images, Signals and Intelligent Systems, University of Paris-Est Crteil, Crteil, France. His research interests include modeling, identification, and control of robotic systems (wearable robots). 